Research Article

\title{
Chaos and Stability of Spur Gear Transmission System for Locomotive Based on Energy Method and Floquet Theory
}

\author{
Junguo Wang ${ }^{D}$, Bo Lv, and Yongxiang Zhao \\ School of Mechanical Engineering, Southwest Jiaotong University, Chengdu 610031, China \\ Correspondence should be addressed to Junguo Wang; jgwang@swjtu.edu.cn
}

Received 22 May 2018; Accepted 11 October 2018; Published 1 November 2018

Academic Editor: Luca Landi

Copyright (C) 2018 Junguo Wang et al. This is an open access article distributed under the Creative Commons Attribution License, which permits unrestricted use, distribution, and reproduction in any medium, provided the original work is properly cited.

Considering the internal and external excitations such as time-varying mesh stiffness (TVMS), backlash, transmission error, torque of the traction motor, and load torque of the wheel/rail, a lumped mass model of the spur gear drive system for a railway locomotive is established. Based on Ma models in the relevant literatures, TVMS is calculated by simplifying a gear tooth as a cantilever beam on the root circle, taking into account the effects of extended tooth contact as well as revised foundation stiffness. The bifurcation diagrams and Lyapunov exponent curves of the model parameters are drawn by the numerical method, and the mechanism of chaos evolution of the gear transmission system is analyzed. According to the Floquet theory, variation curves of the maximum Floquet multiplier with pinion speed and support stiffness ratio are drawn by numerical methods. Combined with the bifurcation diagram of the system, the influences of model parameter on the stability of the system are analyzed, and the evolution laws of periodic motion and bifurcation phenomenon are gained. These research results provide the theoretical evidence of model parameter design of the locomotive transmission system.

\section{Introduction}

As an important device of rotating machinery, the gear transmission system has found extensive applications in many fields such as high-speed locomotive, petrochemical, and power generation industries. Due to the complicated structure, harsh operation environment, and flexible excitations, the geared system is prone to damage to some degree and recognized as the vulnerable part in most cases. At present, there is a growing demand for development of reliable transmission systems, with higher requirements of torque, speed, and compactness. To satisfy these demands, engineers and researchers need to further understand the function of nonlinear phenomena involved in gear transmission. As a consequence, it is imperative to investigate dynamic behavior and evolution mechanism by developing a reliable dynamic model of the gear system [1] and ensure the safe and stable operation for the rotating equipment.

With the development of nonlinear dynamics theory, the nonlinear characteristics of the geared system including stability, bifurcations, and chaos have become the most interesting research areas $[2,3]$. Over the past decades, the characteristic analysis above is practically based on the modelling, and thus, modelling of the gear system is considered as a fundamental problem which is still the research hotspot $[4,5]$. The factors such as backlash [6,7], time-varying mesh stiffness (TVMS) $[8,9]$, and transmission error and lateral-torsional coupled vibration $[10,11]$ will directly affect the dynamics including the stability and reliability of the gear system. Various approaches were successively adopted to illustrate the nonlinear characteristics including stability of the periodic solution, bifurcation, and chaos. In these gear models, TVMS is usually considered a simple sinusoidal function, a Fourier series, or a rectangular waveform function expression. An effective way to evaluate the TVMS is essential to understand the dynamic properties. In general, the finiteelement methods (FEMs) and analytical methods are two main ways to calculate TVMS. FEM is time-consuming because every gear pair requires modelling, while analytical method can offer a general approach to evaluate TVMS. The relative contribution of individual components, such as bending, shear, and Hertzian contact stiffness, can be analyzed separately. Considering various impact factors including extended tooth contact, revised fillet-foundation stiffness, and nonlinear contact stiffness, Ma et al. [12] developed an analytical model for TVMS calculation. Chen and 
Shao [13] proposed a TVMS model with tooth root crack propagating along both tooth width and crack depth. In [14], the potential energy method was applied to evaluate TVMS as a planetary gear set. Based on single-point estimation of TVMS, Wan et al. [15] obtained the TVMS of a helical gear pair and verified the simulated results by the FEM and ISO standard method.

In [16], Wei et al. developed a six-degree-of-freedom dynamic model coupled flexional, torsional, and axial motion of the helical gear transmission system, which includes TVMS, bearing support stiffness, mesh damping, and backlash. The effects on dynamic transmission errors and stabilities by contact ratio, support stiffness, and mesh damping as well as backlash were analyzed. Farshidianfar and Saghafi [17] studied the global homoclinic bifurcation and transition to chaotic behavior of a nonlinear gear system by means of the Melnikov method. The research results of Seyranian et al. [18] show that Floquet theory is more suitable for analyzing the stability of the multiparameter periodic system and multidegree-of-freedom gear system. Based on the nonlinear dynamic model of the three-degree-of-freedom gear system, Liu et al. [19] studied the system stability and the type of bifurcation by Floquet theory and obtained the stable and unstable periodic orbits. Considering TVMS, variable damping, and sliding friction, Vaishya and Singh [20] built a spur gear system model and discussed the stability of the gear transmission system on the basis of Floquet theory.

The gear transmission system of railway locomotive is a kind of typical nonlinear system with a complex structure and service environment. Due to the complex meshing relationship and wheel/rail excitation, the key components of the transmission system are prone to complex dynamic behaviors. At present, the research of chaos and stability is mostly limited to the general mechanical transmission system, and very limited works have addressed the effect on the gear dynamic response for railway locomotive. On account of the harsh operation environment including high speed and heavy load, the driving system of locomotive often bears a high-frequency alternating load generated by single- and double-gear meshing in the process of operation. For this reason, a three-degree-offreedom spur gear drive system model is derived for a typical locomotive, in which the parameters including TVMS, transmission error, mesh damping, and wheel/rail adhesion torque are considered as uncertain but bounded parameters. The effects of those uncertain parameters on the chaos are discussed. And the influences of model parameter changes on the stability of the system are also analyzed on the basis of Floquet theory. Some results are considered as references for the parameters design or vibration control of the gear transmission system for railway locomotive.

\section{Dynamic Model of the Geared System for Locomotive}

2.1. Gear Transmission System Model. As illustrated in Figure 1, the spur gear transmission system is mainly composed of a motor, spur gear pair, and wheel set. The pinion is directly installed on the armature axis of the motor, which provides

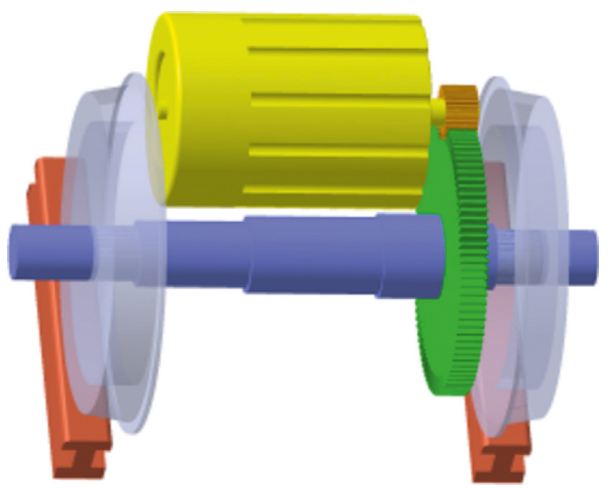

FIgURE 1: Schematic of the spur gear transmission system for railway locomotive.

driving torque of the transmission system, and a gear is installed on the wheel set, which is considered to be the load applied on the geared system. The gear transmission system can be simplified as seen in Figure 2, in which gear meshing is described by backlash, stiffness, and damping elements along the line-of-action (LOA) direction.

Let $m_{1}$ and $m_{2}$ be the mass of the pinion and gear, $I_{1}$ and $I_{2}$ the mass moment of inertia of the pinion and gear, $R_{1}$ and $R_{2}$ the base circle radius of the pinion and gear, $k_{1}$ and $k_{2}$ the bearing support stiffness of the pinion and gear, $c_{1}$ and $c_{2}$ the bearing damping of the pinion and gear, $k(t)$ the timevarying mesh stiffness, $c_{m}$ the damping coefficient of the gear mesh, $y_{1}$ and $y_{2}$ the translational displacement of the pinion and gear, $\theta_{1}$ and $\theta_{2}$ the rotational displacement of the pinion and gear, $T_{1}$ the drive torque provided by the motor under the rated power, and $T_{2}$ the load torque caused by the wheel/rail. Hence, the model of the geared system can be derived as follows:

$$
\left\{\begin{array}{l}
m_{1} \ddot{y}_{1}+c_{1} \dot{y}_{1}+k_{1} y_{1}=F_{0}(t), \\
I_{1} \ddot{\theta}_{1}=T_{1}-R_{1} F_{0}(t), \\
m_{2} \ddot{y}_{2}+c_{2} \dot{y}_{2}+k_{2} y_{2}=-F_{0}(t), \\
I_{2} \ddot{\theta}_{2}=-T_{2}+R_{2} F_{0}(t),
\end{array}\right.
$$

where meshing force $F_{0}(t)=k(t) f\left(R_{1} \theta_{1}-R_{2} \theta_{2}+y_{1}-y_{2}-\right.$ $e(t))+c_{m}\left(R_{1} \theta_{1}-R_{2} \dot{\theta}_{2}+\dot{y}_{1}-\dot{y}_{2}-\dot{e}(t)\right)$. Let $y=R_{1} \theta_{1}-$ $R_{2} \theta_{2}+y_{1}-y_{2}-e(t)$ and $e(t)$ be the static transmission error, then the model above can be rewritten as follows:

$$
\left\{\begin{array}{l}
m_{1} \ddot{y}_{1}+c_{1} \dot{y}_{1}+k_{1} y_{1}=c_{m} \dot{y}+k(t) f(y), \\
m_{2} \ddot{y}_{2}+c_{2} \dot{y}_{2}+k_{2} y_{2}=-c_{m} \dot{y}-k(t) f(y), \\
m_{e} \ddot{y}-m_{e} \ddot{y}_{1}+m_{e} \ddot{y}_{2}+c_{m} \dot{y}+k(t) f(y)=F_{e}(t)+F_{h}(t),
\end{array}\right.
$$

where $\omega$ is the meshing frequency and $m_{\mathrm{e}}$ is the equivalent mass of the gear pair. If the total backlash is $2 b$, introducing natural frequency $\omega_{\mathrm{n}}=\sqrt{k(t) / m_{\mathrm{e}}}$, nondimensional time $\tau=\omega_{\mathrm{n}} t, w_{1}=y_{1} / b, w_{2}=y_{2} / b$, and $w_{3}=y / b$; hence, the model (2) can be simplified as follows:

$$
\left\{\begin{array}{l}
\ddot{w}_{1}+c_{11} \dot{w}_{1}+k_{11} w_{1}=c_{13} \dot{w}+k_{13} f\left(w_{3}\right), \\
\ddot{w}_{2}+c_{22} \dot{w}_{2}+k_{22} w_{2}=-c_{23} \dot{w}_{3}-k_{23} f\left(w_{3}\right), \\
\ddot{w}_{3}-\ddot{w}_{1}+\ddot{w}_{2}+c_{33} \dot{w}_{3}+k_{33} f\left(w_{3}\right)=P_{m}+P_{a} \cos (\Omega \tau) .
\end{array}\right.
$$




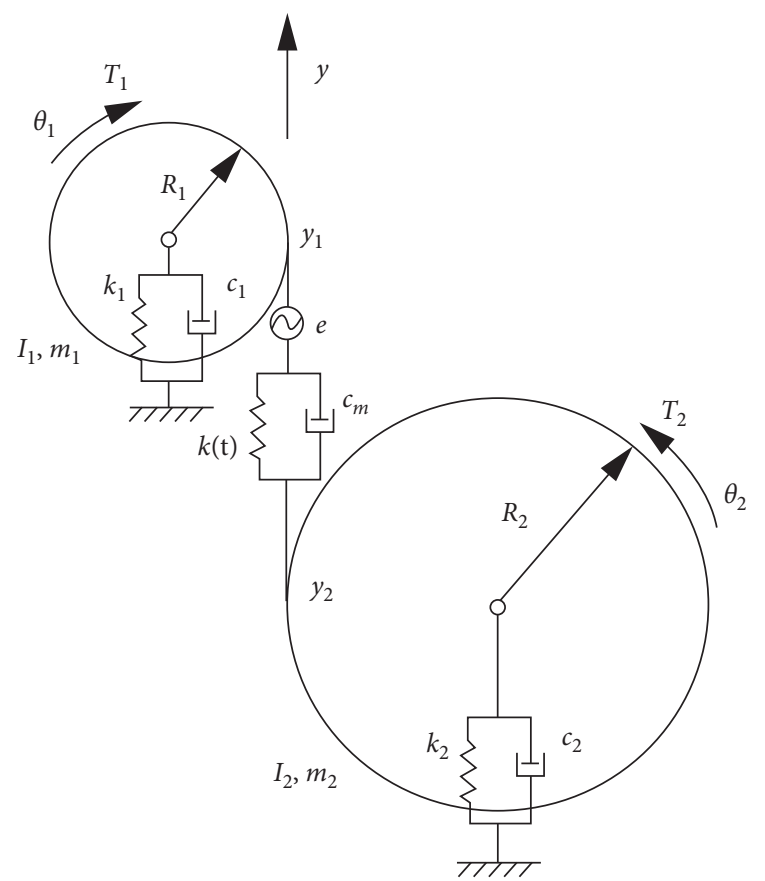

FIgURE 2: Simplified model of the spur gear transmission system for railway locomotive.

The relevant variables are defined as follows:

$$
\begin{aligned}
c_{11} & =\frac{c_{1}}{m_{1} \omega_{n}}, \\
c_{22} & =\frac{c_{2}}{m_{2} \omega_{n}}, \\
c_{33} & =\frac{c_{m}}{m_{e} \omega_{n}}, \\
c_{13} & =\frac{c_{m}}{m_{1} \omega_{n}}, \\
c_{23} & =\frac{c_{m}}{m_{2} \omega_{n}}, \\
k_{11} & =\frac{k_{1}}{m_{1} \omega_{n}^{2}}, \\
k_{22} & =\frac{k_{2}}{m_{2} \omega_{n}^{2}}, \\
k_{33} & =\frac{k(t)}{m_{e} \omega_{n}^{2}}, \\
P_{a} & =\frac{e_{a} \Omega^{2}}{b}, \\
k_{23} & =\frac{k_{m}}{m_{e} \omega_{n}^{2}}, \\
F_{m} & \left.=\frac{c_{m}\left(R_{1}\right.}{b k_{1}},-R_{2} \dot{\theta}_{2}+\dot{y}_{1}-\dot{y}_{2}-\dot{e}\right), \\
0, & -w_{3}, \quad w_{3}<-1 .
\end{aligned}
$$

2.2. Wheel/Rail Adhesion Torque. To express the slippage in a more physical sense, the slip rate of locomotive transmission dynamics can be defined as follows [21]:

$$
s=\frac{\left(v-\omega_{w} R\right)}{v},
$$

where $v$ is the instantaneous speed of the wheel set, $\omega_{w}$ the instantaneous angular velocity, and $R$ the radius of the wheel. If the number of gear teeth is $Z_{2}, \omega_{w}=2 \pi \omega / Z_{2}$. The wheel/ rail tangential force coefficient is inversely related to the slip after the peak of adhesion, and the simplified wheel/rail adhesion curve can be indicated by a sectional linear function [21], as shown in Figure 3:

$$
F= \begin{cases}\frac{f_{\mathrm{m}} s}{s_{\mathrm{c}}}, & 0 \leq s \leq s_{\mathrm{c}} \\ f_{\mathrm{m}}+k_{f}\left(s-s_{\mathrm{c}}\right), & s_{\mathrm{c}}<s\end{cases}
$$

where $f_{\mathrm{m}}$ is the maximum tangential force coefficient, $s_{\mathrm{c}}$ the critical slip rate, and $k_{f}$ the negative slope on sliding. For the simplified model, the adhesion state under positive slope is considered to stick, the adhesion state under negative slope is considered to slip, but the dispersion of adhesion is not considered. When the motor runs at rated power, $s_{\mathrm{c}}$ is 0.01 , and $s$ is changed from 0 to 0.03 .

Let the axle load of locomotive be $m g$, the wheel/rail adhesion torque can be obtained as follows:

$$
T_{2}=F_{r} R= \begin{cases}\frac{m g R f_{\mathrm{m}}}{s_{\mathrm{c}}}, & 0 \leq s \leq s_{\mathrm{c}}, \\ m g R f_{\mathrm{m}}+m g R k_{f}\left(s-s_{\mathrm{c}}\right), & s_{\mathrm{c}}<s .\end{cases}
$$

\section{Calculation of Time-Varying Mesh Stiffness}

The mesh stiffness is a time-varying parameter, as the number of engaged teeth varies according to the contact ratio. Considering the misalignment of the gear root circle and base circle, Ma et al. [22] proposed a TVMS model for a healthy gear pair; thereafter, they presented an improved method for mesh stiffness of spur gears with tip relief $[23,24]$. Based on ANSYS software, Wang [25] presented an approach to predict TVMS by establishing contact elements. Saxena et al. [26] developed a method to calculate TVMS for different spall shapes, sizes, and locations. In the traditional method, the gear tooth is mostly modelled as a nonuniform cantilever beam on the base circle. In this study, in order to improve the analytical method, considering the effects of extended tooth contact and revised foundation stiffness, an improved analytical TVMS calculation method is given according to Ma models and methods above.

3.1. Determination of the Tooth Profile Model. Deflections of a spur gear tooth can be determined by considering it as a nonuniform cantilever beam with an effective length $d$ displayed in Figure 4. Here, $r_{\text {int }}$ is the radius of the gear inside holes, $r_{c}$ the radius of the tip fillet, $\beta$ the operating pressure angle, $\theta_{b}$ the half tooth angle on the base circle of the gear, $\theta_{c}$ the angle corresponding to the point $c, x_{\beta}$ the distance between contact point and central line of the tooth, and $y_{\beta}$ the distance between contact point and original point: 


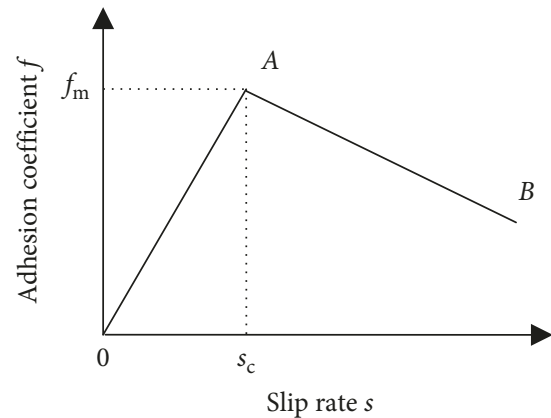

(a)

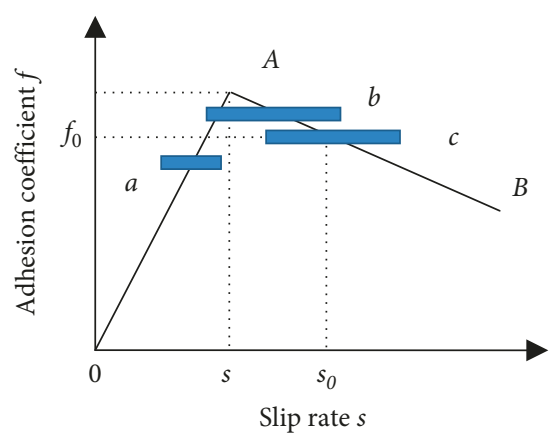

(b)

FIgURE 3: (a) Simplified curve of the tangential force coefficient of locomotive. (b) Mechanism curve of slippery vibration.

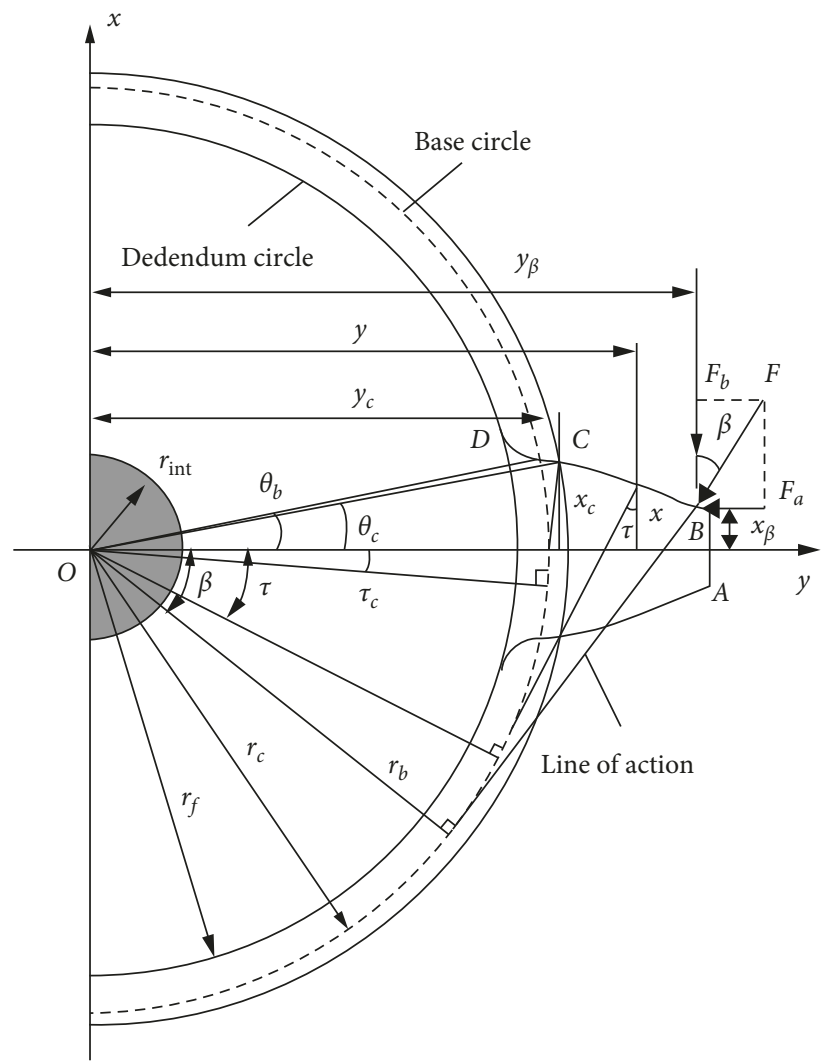

FIgURE 4: Modelling of the spur gear tooth as a nonuniform cantilever beam.

$$
\left\{\begin{array}{l}
r_{b}=\frac{m Z \cos \alpha}{2} \\
r_{f}=\frac{m Z}{2-\left(h_{a}^{*}+c^{*}\right) m}
\end{array}\right.
$$

For an involute curve, there exists the following geometric relationship:

$$
\left\{\begin{array}{l}
x=r_{i} \sin \varphi \\
y=r_{i} \cos \varphi \\
\varphi=\frac{\pi}{2 Z-\left(\operatorname{inv} \alpha_{i}-\operatorname{inv} \alpha\right)}
\end{array}\right.
$$

The transition curve is the tooth profile between involute starting point and root circle, and it is cut out by the cutter tip. Hence, the transition curve equations can be written as follows:

$$
\left\{\begin{array}{l}
x=r \sin \psi-\left(\frac{a_{1}}{\sin \gamma+r_{\rho}}\right) \cos (\gamma-\psi), \\
y=r \cos \psi-\left(\frac{a_{1}}{\sin \gamma+r_{\rho}}\right) \sin (\gamma-\psi), \\
\psi=\frac{\left(a_{1} / \tan \gamma+b_{1}\right)}{r}
\end{array}\right.
$$


and

$$
\left\{\begin{array}{l}
a_{1}=\left(h_{a}^{*}+c^{*}\right) m-r_{\rho}, \\
r_{\rho}=c^{*} \times \frac{m}{(1-\sin \alpha)}, \quad \alpha \leq \gamma \leq \pi / 2, \\
b_{1}=\frac{\pi m}{4+h_{a}^{*} m \tan \alpha+r_{\rho} \cos \alpha} .
\end{array}\right.
$$

3.2. An Energy Method for TVMS Calculation. Based on elastic mechanics, Hertzian contact stiffness can be written as follows [23-27]:

$$
\left\{\begin{array}{l}
k_{h}=\frac{\pi E L}{4\left(1-v^{2}\right)}, \\
k_{f}=\frac{E L}{\cos ^{2} \beta\left[L^{*}\left(u_{f} / S_{f}\right)^{2}+M^{*}\left(u_{f} / S_{f}\right)+P\left(1+Q^{*} \tan ^{2}\right) \beta\right]},
\end{array}\right.
$$

where the parameters $u_{f}, S_{f}, L^{*}, M^{*}, P^{*}$, and $Q^{*}$ can be found in [27]. The Hertzian energy, bending, shear, fillet foundation, and axial compressive energies can be written as follows [22]:

$$
\begin{gathered}
U_{h}=\frac{F^{2}}{2 k_{h}}, \\
U_{b}=\frac{F^{2}}{2 k_{b}}, \\
U_{s}=\frac{F^{2}}{2 k_{s}}, \\
U_{f}=\frac{F^{2}}{2 k_{f}}, \\
U_{a}=\frac{F^{2}}{2 k_{a}} .
\end{gathered}
$$

Based on the beam theory, the potential energy stored in a meshing gear tooth is obtained by the following equation [22]:

$$
\left\{\begin{array}{l}
U_{b}=\int_{y_{D}}^{y_{C}}\left(\frac{M_{1}^{2}}{2 E I_{y 1}}\right) d y_{1}+\int_{y_{C}}^{y_{\beta}}\left(\frac{M_{2}^{2}}{2 E I_{y 2}}\right) d y_{2}, \\
U_{s}=\int_{y_{D}}^{y_{C}}\left(\frac{1.2 F_{b}^{2}}{2 G A_{y 1}}\right) d y_{1}+\int_{y_{C}}^{y_{\beta}}\left(\frac{1.2 F_{b}^{2}}{2 G A_{y 2}}\right) d y_{2}, \\
U_{a}=\int_{y_{D}}^{y_{C}}\left(\frac{F_{a}^{2}}{2 E A_{y 1}}\right) d y_{1}+\int_{y_{C}}^{y_{\beta}}\left(\frac{F_{a}^{2}}{2 E A_{y 2}}\right) d y_{2},
\end{array}\right.
$$

and

$$
\left\{\begin{array}{l}
M_{1}=F_{b}\left(y_{\beta}-y_{1}\right)-F_{a} x_{\beta}, \\
M_{2}=F_{b}\left(y_{\beta}-y_{2}\right)-F_{a} x_{\beta}, \\
G=\frac{E}{2(1+v)}, \\
x_{\beta}=r_{b}\left[\left(\beta+\theta_{b}\right) \cos \beta-\sin \beta\right], \\
y_{\beta}=r_{b}\left[\left(\beta+\theta_{b}\right) \sin \beta+\cos \beta\right],
\end{array}\right.
$$

$$
\left\{\begin{array}{l}
I_{y 1}=\frac{\left(2 x_{1}\right)^{3} L}{12}, \\
I_{y 2}=\frac{\left(2 x_{2}\right)^{3} L}{12}, \\
A_{y 1}=2 x_{1} L, \\
A_{y 2}=2 x_{2} L, \\
x_{1}=r \sin \psi-\left(\frac{a_{1}}{\sin \gamma+r_{\rho}}\right) \cos (\gamma-\psi), \\
x_{2}=r_{b}\left[\left(\tau+\theta_{b}\right) \cos \tau-\sin \tau\right], \\
y_{1}=r \cos \psi-\left(\frac{a_{1}}{\sin \gamma+r_{\rho}}\right) \sin (\gamma-\psi), \\
y_{2}=r_{b}\left[\left(\tau+\theta_{b}\right) \sin \tau+\cos \tau\right],
\end{array}\right.
$$

where $M_{1}$ and $M_{2}$ are the bending moments of $F_{b}$ and $F_{a}$ on the part of transition and involute curves, $y_{1}$ and $y_{2}$ the horizontal coordinates, and $G$ the shear modulus. Hence, the bending, shear, and axial compressive stiffness can be obtained as follows:

$$
\left\{\begin{array}{c}
\frac{1}{k_{b}}=\int_{\pi / 2}^{\alpha} \frac{\left[\cos \beta\left(y_{\beta}-y_{1}\right)-x_{\beta} \sin \beta\right]^{2}}{E I_{y 1}} \frac{d y_{1}}{d \gamma} d \gamma \\
+\int_{\tau_{c}}^{\beta} \frac{\left(\cos \beta\left(y_{\beta}-y_{2}\right)-x_{\beta} \sin \beta\right)^{2}}{E I_{y 2}} \frac{d y_{2}}{d \tau} d \tau, \\
\frac{1}{k_{s}}=\int_{\pi / 2}^{\alpha} \frac{1.2 \cos ^{2} \beta}{G A_{y 1}} \frac{d y_{1}}{d \gamma} d \gamma+\int_{\tau_{c}}^{\beta} \frac{1.2 \cos ^{2} \beta}{G A_{y 2}} \frac{d y_{2}}{d \tau} d \tau, \\
\frac{1}{k_{a}}=\int_{\pi / 2}^{\alpha} \frac{\sin ^{2} \beta}{E A_{y 1}} \frac{d y_{1}}{d \gamma} d \gamma+\int_{\tau_{c}}^{\beta} \frac{\sin ^{2} \beta}{E A_{y 2}} \frac{d y_{2}}{d \tau} d \tau,
\end{array}\right.
$$

with 


$$
\left\{\begin{aligned}
\frac{d y_{1}}{d \gamma}= & \frac{a_{1} \sin \psi\left(1+\tan ^{2} \gamma\right)}{\tan ^{2} \gamma}+\frac{a_{1} \cos \gamma \sin (\gamma-\psi)}{\sin ^{2} \gamma} \\
& -\left(\frac{a_{1}}{\sin \gamma}+r_{\rho}\right) \cos (\gamma-\psi)\left(1+\frac{a_{1}\left(1+\tan ^{2} \gamma\right)}{r \tan ^{2} \gamma}\right) \\
\frac{d y_{2}}{d \tau}= & r_{b}\left(\tau+\theta_{b}\right) \cos \tau .
\end{aligned}\right.
$$

Let subscripts 1 and 2 be pinion and gear, the singletooth-pair mesh stiffness can be defined as follows [23, 24]:

$$
k^{i}=\frac{1}{\left(1 / k_{h}+1 / k_{t 1}+1 / k_{f 1}+1 / k_{t 2}+1 / k_{f 2}\right)},
$$

and

$$
k_{t}=\frac{1}{\left(1 / k_{b}+1 / k_{s}+1 / k_{a}\right)} \text {. }
$$

If $N$ is the number of meshing tooth pairs, the TVMS can be calculated as follows:

$$
k=\sum_{i=1}^{N} k^{i}
$$

However, in fact, two meshing tooth pairs share the one gear body, which leads to a mesh stiffness higher than the actual value in double-tooth engagement. Thus, an improved method is adopted where the total mesh stiffness is expressed as follows $[23,24]$ :

$$
\begin{aligned}
k= & \frac{1}{\left(1 / \lambda_{1 N} k_{f 1}+1 / k_{\text {tooth }}+1 / \lambda_{2 N} k_{f 2}\right)} \\
& \left(\lambda_{11}=\lambda_{21}=1, \text { when } N=1\right), \\
k_{\text {tooth }}= & \sum_{i=1}^{N} k_{\text {tooth }}^{i}, \\
k_{\text {tooth }}^{i}= & \frac{1}{1 / k_{h i}+1 / k_{t 1}+1 / k_{t 2}},
\end{aligned}
$$

where $\lambda$ represents the correction coefficient of the filletfoundation stiffness, $k_{\text {tooth }}$ the total mesh stiffness for $N$ meshing tooth pairs, and $k_{\text {tooth }}^{i}$ the mesh stiffness of the $i$ th tooth pair.

The correction coefficients of the fillet-foundation stiffness need to be determined using the FE method, and the detailed calculation is given in $[23,24]$. Hence, the stiffness caused by tooth deformation during double-tooth engagement can be calculated by the following equation:

$$
k_{\text {tooth }}=\frac{F}{E_{r}^{2}-E_{P}^{i}} \text { with } E_{p}^{i}=\min \left(E_{p}^{1}, E_{p}^{2}\right) \text {. }
$$

The total mesh stiffness can be obtained from Equation (20). In addition, mesh stiffness of a single-tooth pair is a function of tooth contact deformation $E_{\mathrm{d}}$ and meshing position $j$, which can be expressed as follows $[23,24]$ :

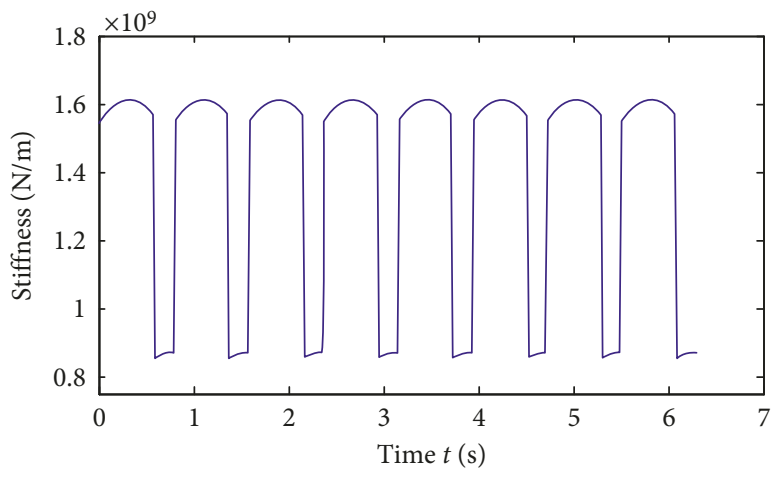

FIgURE 5: The curve of mesh stiffness in a mesh period.

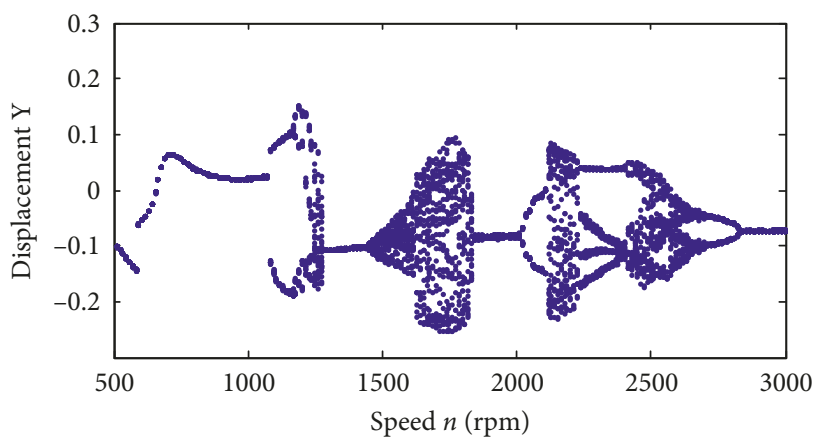

Figure 6: Bifurcation diagram of displacement with the pinion speed changes.

$$
k= \begin{cases}k^{i}, & E_{\mathrm{d}}^{i}>0, \\ 0, & E_{\mathrm{d}}^{i} \leq 0,\end{cases}
$$

where the calculation of correction coefficients is shown in the relevant literatures.

The parameters of the gear pair are chosen as follows: the number of teeth on the pinion and gear is 23 and 120 , radius of the pinion and gear is 86 and $450 \mathrm{~mm}$, module is $8 \mathrm{~mm}$, teeth width is $140 \mathrm{~mm}$, coincidence degree is 1.7315 , elastic modulus is $209 \mathrm{GPa}$, and Poisson's ratio is 0.3. The TVMS curve which changes with dimensionless time is displayed in Figure 5. With the help of this figure, the curve-fitted stiffness equation is derived as follows:

$k(\tau)= \begin{cases}1.55 \times 10^{9}+5.31 \times 10^{8} \tau-1.06 \times 10^{9} \tau^{2}, & 0<\tau \leq 1.463 \pi, \\ 5.72 \times 10^{8}+9.93 \times 10^{8} \tau-8.25 \times 10^{8} \tau^{2}, & 1.463 \times \pi<\tau \leq 2 \pi .\end{cases}$

\section{Chaos and Stability Analysis of Locomotive Gear Transmission System}

In this section, a case study is analyzed to demonstrate the effect of model parameters on the chaos and stability responses. For the subsequent numerical simulations, some parameters are as follows: $k_{1}=4.5 \times 10^{9} \mathrm{~N} / \mathrm{m}, k_{2}=9 \times$ $10^{9} \mathrm{~N} / \mathrm{m}, c_{1}=c_{2}=500 \mathrm{Ns} / \mathrm{m}, k_{33}(\tau)=k(\tau)$, and $c_{33}=0.05$. 


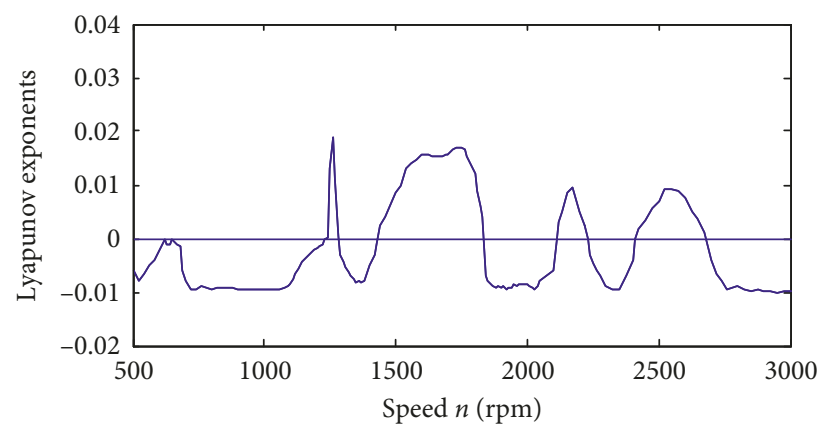

FIGURE 7: Lyapunov exponent diagram of the system with the gear speed changes.

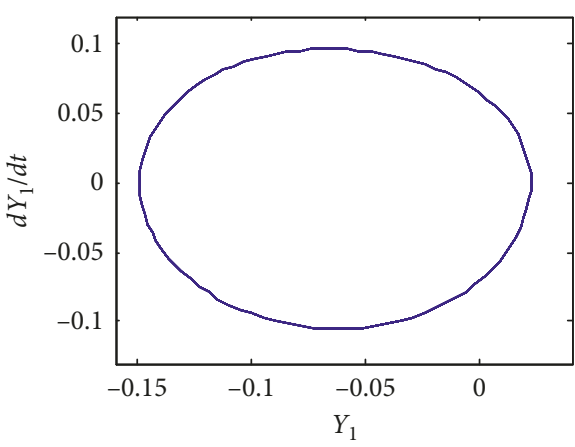

(a)

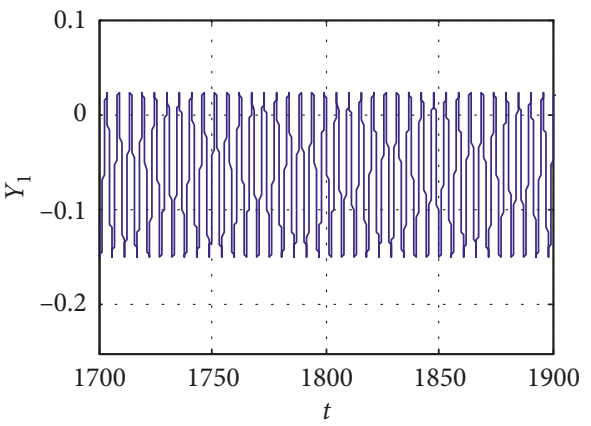

(c)

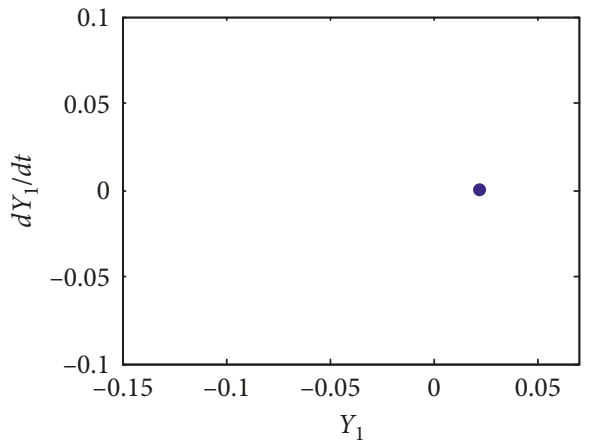

(b)

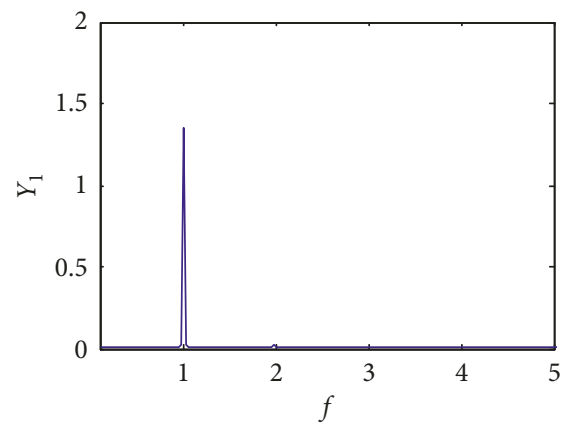

(d)

Figure 8: The dynamic characteristic curve of the system at $\Omega=1000 \mathrm{rpm}$. (a) Phase-plane diagram. (b) Poincaré map. (c) Time-domain response diagram. (d) Amplitude-frequency spectrum.

4.1. Effect of Pinion Speed on Chaotic Characteristics. Figure 6 shows the bifurcation diagram of the gear system in dimensionless displacement using the pinion speed $\Omega$ as the control parameter. It can be seen that various kinds of periodic, multiperiodic, and chaotic responses exist in the system. When $\Omega$ is less than $1070 \mathrm{rpm}$, the dynamic behavior is synchronous with periodic-1 motion; subsequently, it comes into periodic- 2 and periodic- 4 motion at district of (1080-1160) and (1170-1240) rpm, respectively. After a short chaos at district of (1250-1270) rpm, the system enters periodic-1 motion of (1280-1430) rpm. With the increasing pinion speed, it enters chaos at district of (1440-1830) rpm. Thereafter, it comes into periodic-1 and periodic-2 motion between (1840-1990) and (2000-2110) rpm, respectively, and then it enters chaos again within (2120-2220) rpm. When $\Omega$ is higher than $2230 \mathrm{rpm}$, the system enters periodic- 4 , chaotic, and periodic- 2 motion in sequence, in which the intervals are (2230-2400), (24102670), and (2680-2830) rpm, respectively. Once $\Omega$ is higher than $2840 \mathrm{rpm}$, the gear system eventually returns to the periodic- 1 motion state.

The Lyapunov exponent is an important parameter for studying and determining whether the dynamic system is chaotic motion. Figure 7 presents the Lyapunov exponent diagram of the geared system when pinion speed is changed from (500-3000) rpm. It is obvious that the Lyapunov exponents are greater than zero when chaos emerges in the same region of speed change. To accurately determine chaotic motion of the geared system, the phase-plane diagrams, Poincaré maps, time-domain response diagrams, and amplitude-frequency spectrums are listed to illustrate dynamic characteristics of the system in Figures 8-10. 


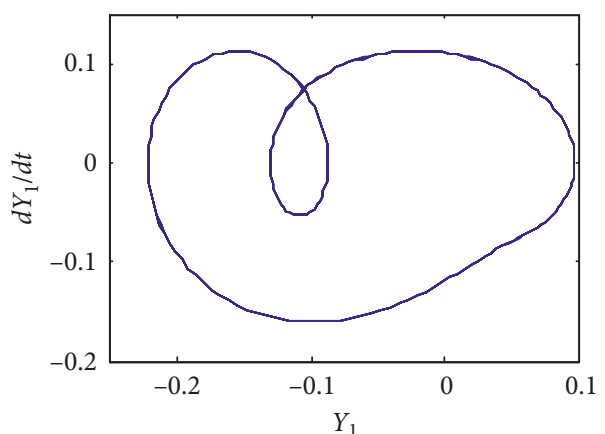

(a)

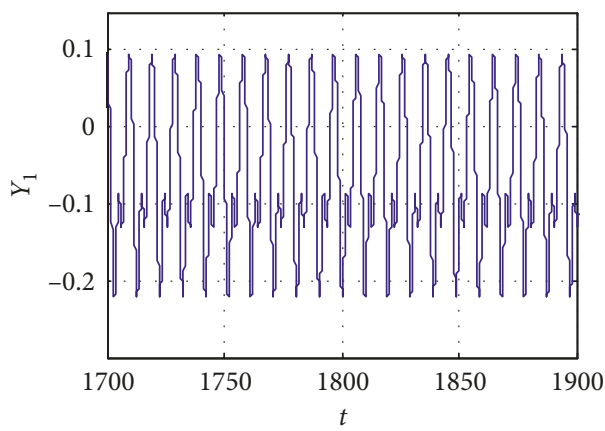

(c)

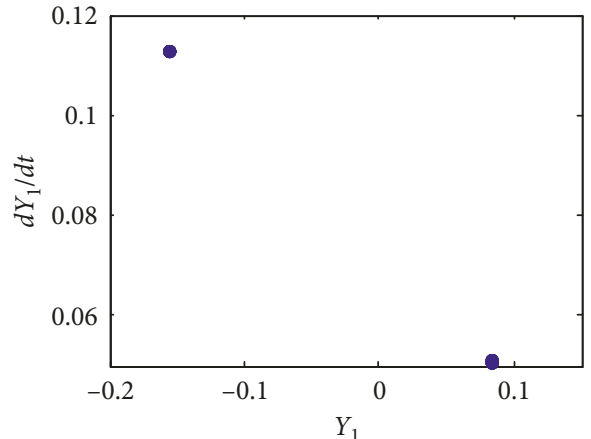

(b)

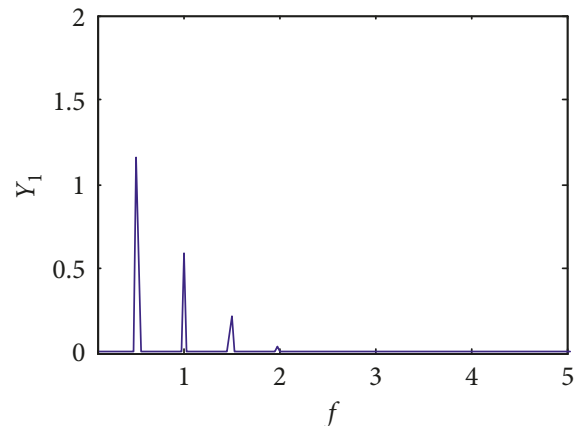

(d)

Figure 9: The dynamic characteristic curve of the system at $\Omega=1150 \mathrm{rpm}$. (a) Phase-plane diagram. (b) Poincaré map. (c) Time-domain response diagram. (d) Amplitude-frequency spectrum.

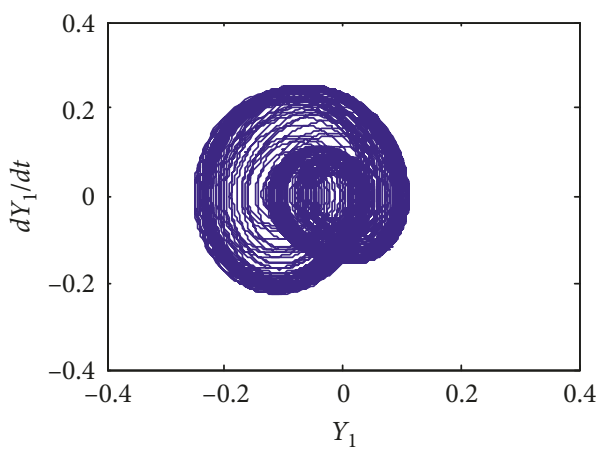

(a)

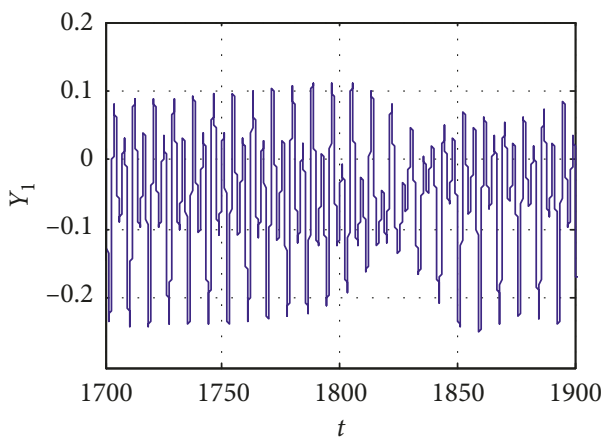

(c)

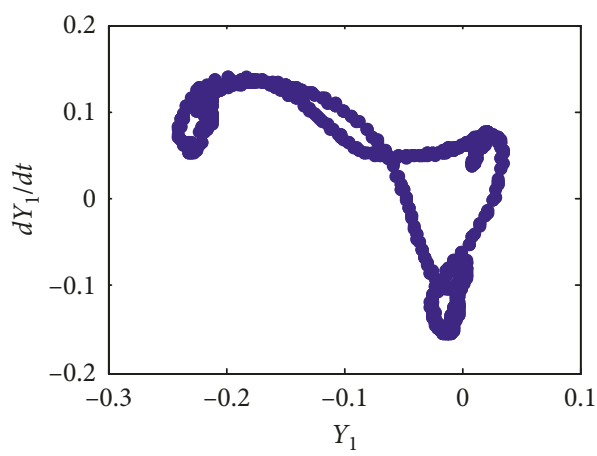

(b)

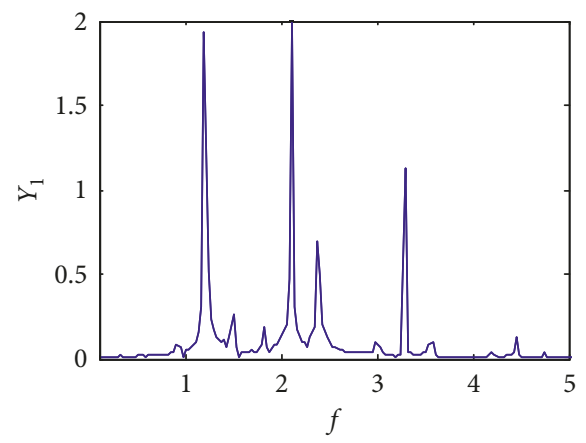

(d)

Figure 10: The dynamic characteristic curve of the system at $\Omega=1600 \mathrm{rpm}$. (a) Phase-plane diagram. (b) Poincaré map. (c) Time-domain response diagram (d) Amplitude-frequency spectrum. 


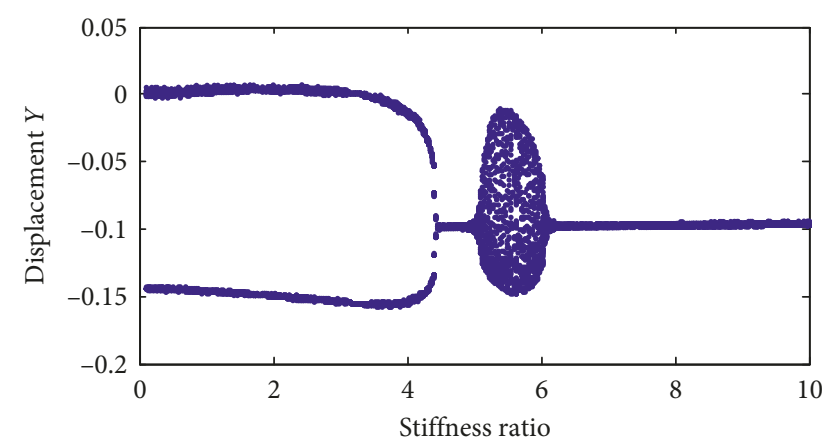

FIGURE 11: Bifurcation diagram of the displacement with support stiffness ratio changes.

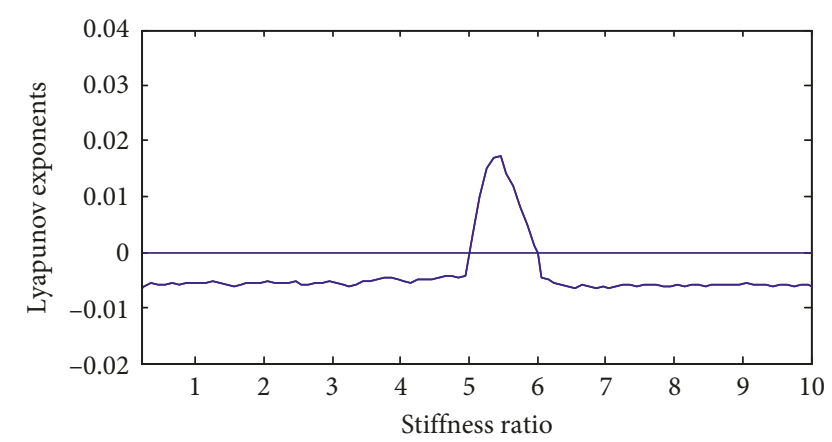

FIGURE 12: Lyapunov exponent diagram of the system with support stiffness ratio changes.

When $\Omega=1000 \mathrm{rpm}$, there is a single point in the Poincaré map, the phase-plane diagram is only one closed circle, the time-domain response diagram shows a sine wave, and the amplitude-frequency spectrum has one-peak amplitude. It means that the system has periodic-1 motion. When $\Omega=1150$, there are two closed circles in the phaseplane diagram, the Poincaré map shows two isolated points, and the amplitude-frequency spectrum has harmonics $1 / 2,2 / 2$, and $3 / 2$, signifying the presence of a period2 unstable ghost attractor, as indicated in Figure 9. When $\Omega=1600 \mathrm{rpm}$, it can be seen that the phase-plane diagram is disordered, the Poincaré map shows many discrete points, the time-domain response diagram also shows nonperiodic motion, and the amplitude-frequency spectrum is continuous. All the dynamic characteristics reveal that the system is in a state of chaos.

\subsection{Effect of Support Stiffness Ratio on Chaotic Characteristics.} Support stiffness of the pinion and gear is the key parameter that affects the dynamic behavior of the gear system. To study the effects, support stiffness ratio $K$, namely, support stiffness of the pinion to gear, is incorporated in the paper, where support stiffness of the pinion $k_{1}=4.5 \times 10^{9} \mathrm{~N} / \mathrm{m}$. Figure 11 shows the bifurcation diagram of displacement using the support stiffness ratio $K$ as a bifurcation parameter. When $K$ is less than 4.5 , the system is in the state of periodic2 motion; after a short periodic-1 movement, the system enters chaotic motion at the district of 5-6. Once $K$ is higher than 6 , the gear transmission system returns to synchronous vibration with period-1.

Figure 12 also shows the Lyapunov exponent diagram of the geared system when the support stiffness ratio $K$ is changed from 1 to 10, in which Lyapunov exponents are greater than zero in the same region compared to the bifurcation diagram, and the chaotic regions are basically the same as those in Figure 11. To accurately determine chaotic motion of the proposed system, the dynamic characteristic curves are also listed in Figures 13-15. The dynamic characteristics have obvious chaotic characteristics as seen in these figures, so the gear system is in chaotic motion in these stiffness ranges.

\subsection{Stability Analysis of Locomotive Gear Transmission System}

4.3.1. Floquet Stability Theory. For the perturbation equation of a nonlinear system [15],

$$
\left\{\begin{array}{l}
\dot{X}=A(t) X, \\
A(t+T)=A(t),
\end{array}\right.
$$

where $T$ represents a system of periodic motion and $A(t+T)$ is the Jacobi matrix of the periodic solution of the steady state. Let $X(t)$ be the basic solution matrix of the equation, there is a constant matrix $D$ and a nonsingular periodic $T$ matrix $\varphi(t)=\varphi(t+T)$ and $X(t)=\varphi(t) \cdot \exp (t \cdot D)$.

Since $d X(t) / d t=A(t) X(t)$, it follows that

$$
\frac{d X(t+T)}{d t}=A(t+T) X(t+T)=A(t) X(t+T)
$$

and

$$
\begin{aligned}
X(t+T) & =\varphi(t+T) \cdot \exp ((t+T) \cdot D) \\
& =\varphi(t) \cdot \exp (t \cdot D) \cdot \exp (T \cdot D) \\
& =X(t) \cdot \exp (T \cdot D),
\end{aligned}
$$

where the constant matrix $\exp (T \cdot D)$ is called the system state transfer matrix, and the eigenvalue of the matrix is called the Floquet multiplier $\lambda_{i}$.

The research on the stability of periodic motion of nonlinear systems can be carried out in the following steps:

(1) Transform differential equation of the original model into a matrix form:

$$
\{\ddot{x}\}+[c(t)]\{\dot{x}\}+[k(t)]\{\ddot{x}\}=\{F(t)\} .
$$

(2) Transform the matrix equation into the state equation form:

$$
\left\{\begin{array}{l}
\{y\}=\left\{\begin{array}{l}
\{x\}, \\
\{\dot{x}\},
\end{array}\right. \\
\{\dot{y}\}=[A(t)]\{y\}+\{B(t)\},
\end{array}\right.
$$




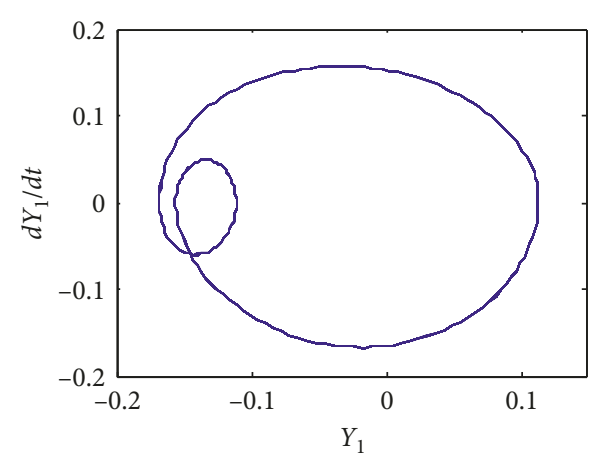

(a)

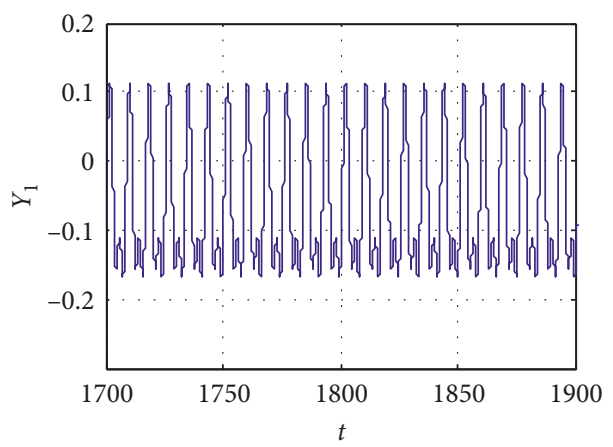

(c)

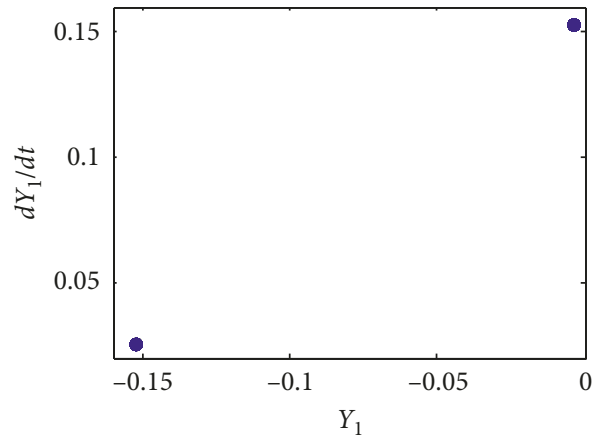

(b)

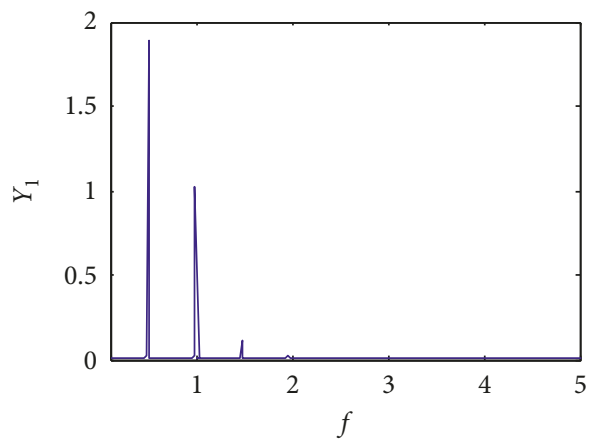

(d)

FIgURE 13: The dynamic characteristic curve of the system at support stiffness ratio $K=2.5$. (a) Phase-plane diagram. (b) Poincaré map. (c) Time-domain response diagram. (d) Amplitude-frequency spectrum.

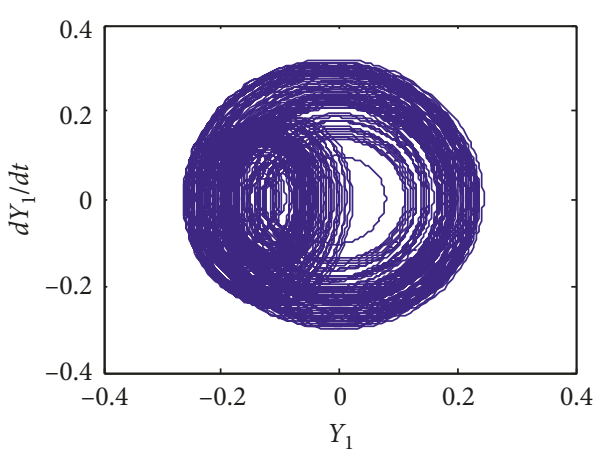

(a)

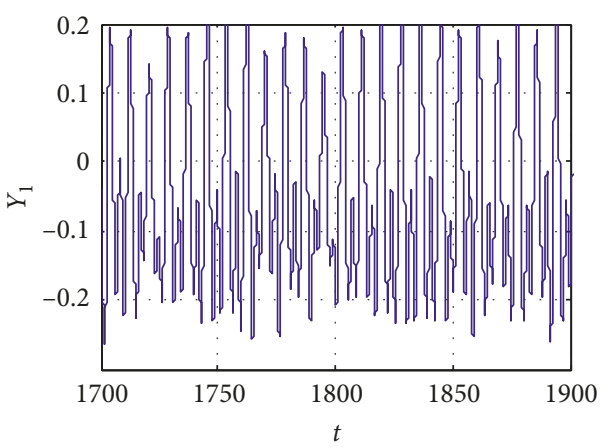

(c)

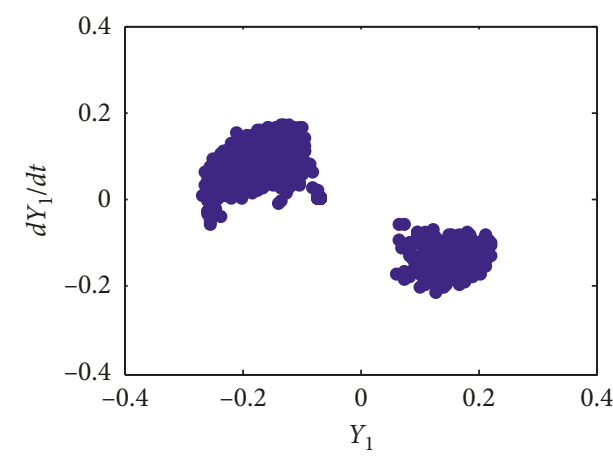

(b)

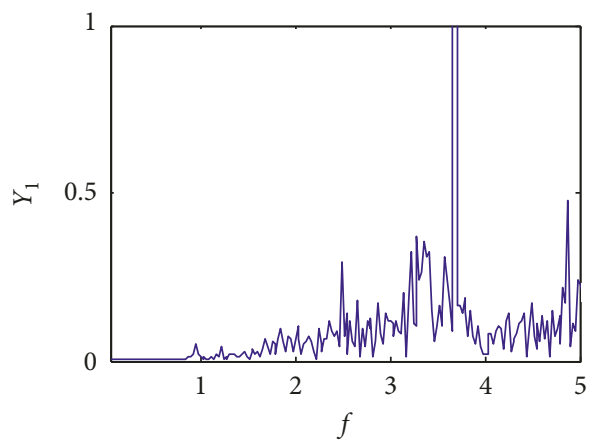

(d)

FIgURE 14: The dynamic characteristic curve of the system at support stiffness ratio $K=5.5$. (a) Phase-plane diagram. (b) Poincaré map. (c) Time-domain response diagram. (d) Amplitude-frequency spectrum. 


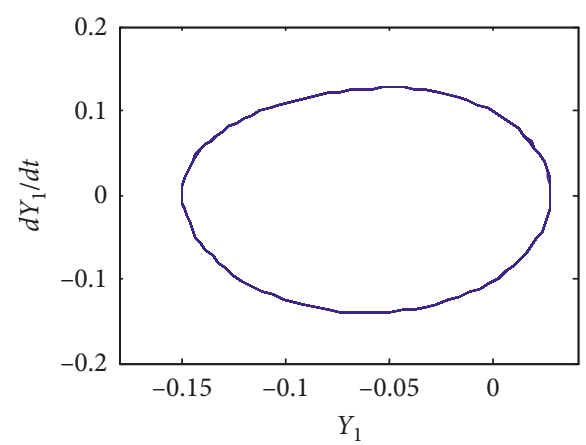

(a)

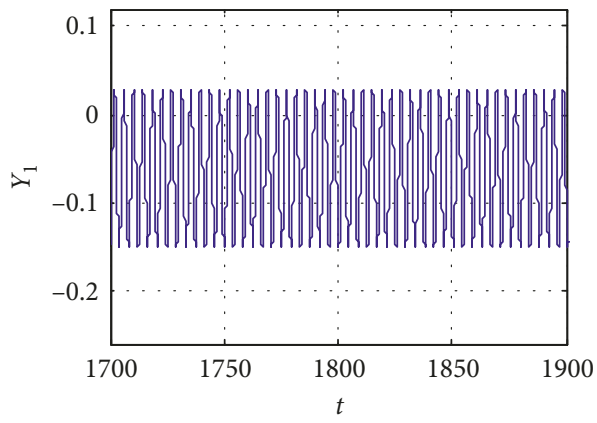

(c)

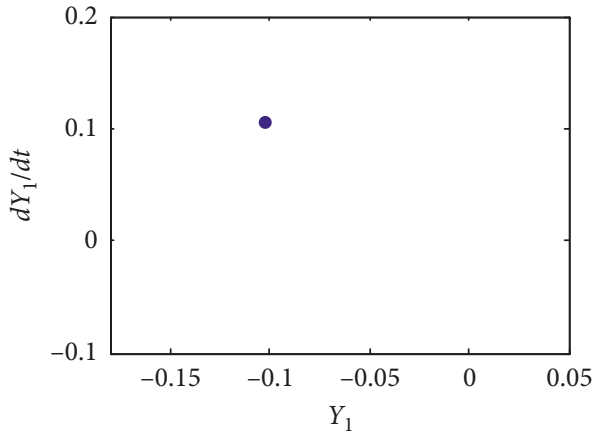

(b)

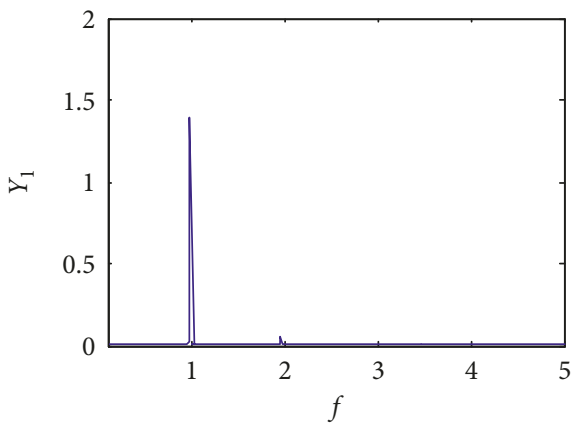

(d)

Figure 15: The dynamic characteristic curve of the system at support stiffness ratio $K=7.5$. (a) Phase-plane diagram. (b) Poincaré map. (c) Time-domain response diagram. (d) Amplitude-frequency spectrum.

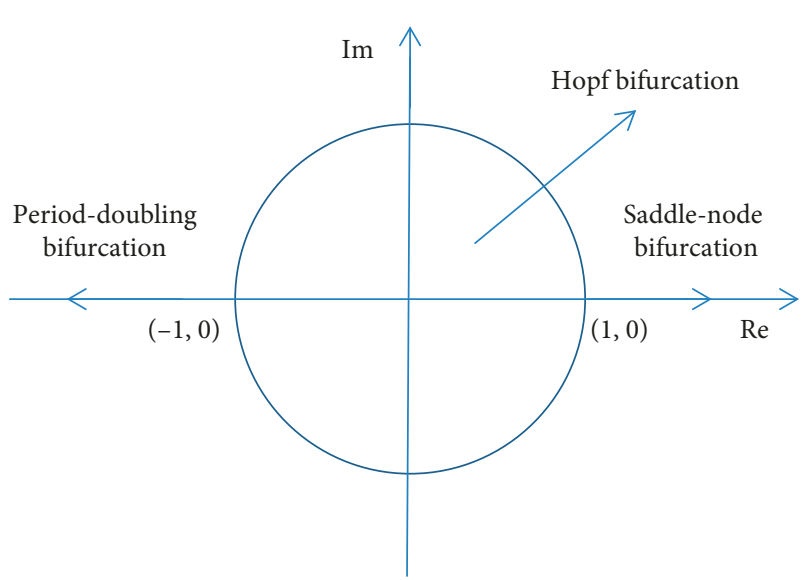

FIGURE 16: Schematic of the Floquet multiplier to generate bifurcation.

with

$$
\begin{aligned}
& {[A(t)]=\left[\begin{array}{cc}
{[0]} & {[I]} \\
-[k(t)] & -[c(t)]
\end{array}\right],} \\
& \{B(t)\}=\left\{\begin{array}{c}
\{0\} \\
\{F(t)\}
\end{array}\right\} .
\end{aligned}
$$

(3) The value of the following matrix equation at periodic $T$ is solved by the numerical integration method:

$$
\left\{\begin{array}{l}
\{\dot{y}\}=[A(t)]\{y\}, \\
\{y(0)\}=[I]_{n} .
\end{array}\right.
$$

(4) The Floquet multiplier of the state transition matrix is obtained from the QR algorithm based on the eigenvalue of the matrix.

(5) According to the location of the Floquet multiplier relative to the unit circle displayed in Figure 16, the stability and bifurcation characteristics of the system can be judged as follows [28]:

(i) When $\left|\lambda_{i}\right|<1(i=1,2, \ldots, n)$, the stable periodic solution is asymptotically stable.

(ii) If there is $\lambda_{j}$ which passes the unit circle outwards through the point of -1 and other $\left|\lambda_{i}\right|<1$ $(i=1,2, \ldots, n, i \neq j)$, the stable periodic solution will have period-doubling bifurcation.

(iii) If there is $\lambda_{j}$ which passes the unit circle outwards through the point of +1 and other $\left|\lambda_{i}\right|<1(i=1,2, \ldots, n, i \neq j)$, the stable periodic solution will have the saddle-node bifurcation.

(iv) If there is a pair of conjugate complex characteristic multipliers, $\lambda_{j}=a \pm j b$, which passes the unit circle outward, and other $\left|\lambda_{i}\right|<1 \quad(i=1$, $2, \ldots, n, i \neq j$ ), the stable periodic solution will have the Hopf bifurcation, and the bifurcation will lead to an invariant torus. 


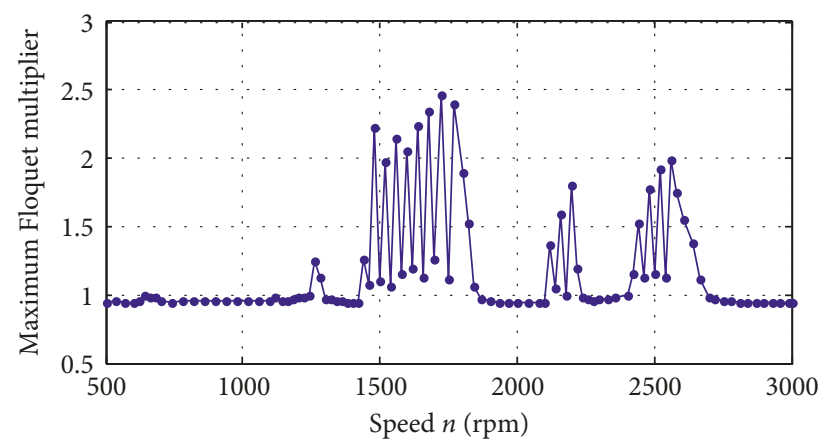

FIGURE 17: Diagram of the maximum Floquet multiplier with pinion speed changes.

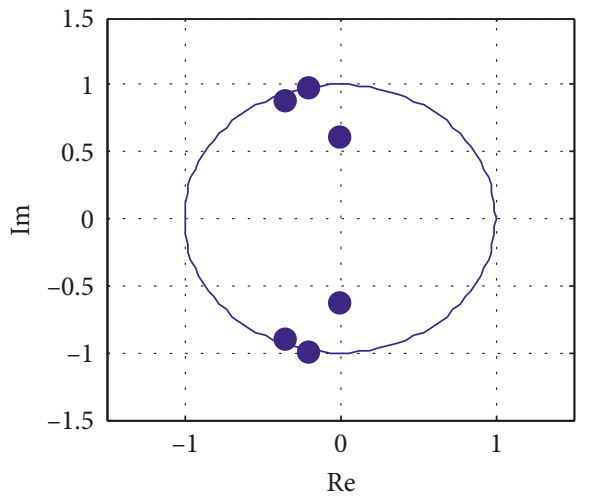

(a)

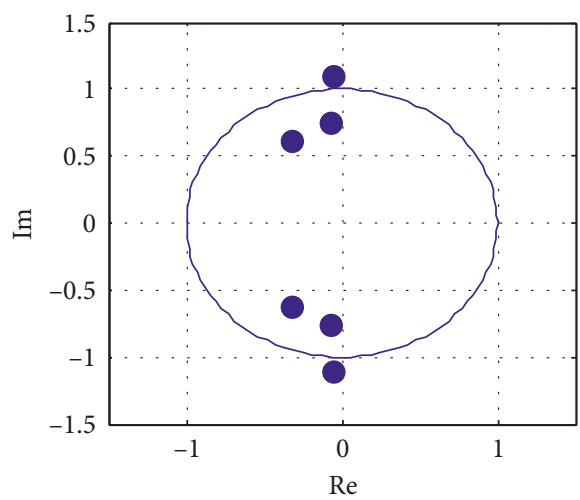

(c)

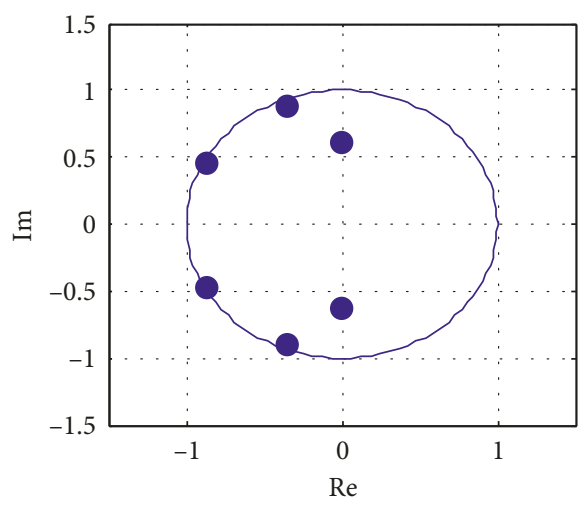

(e)

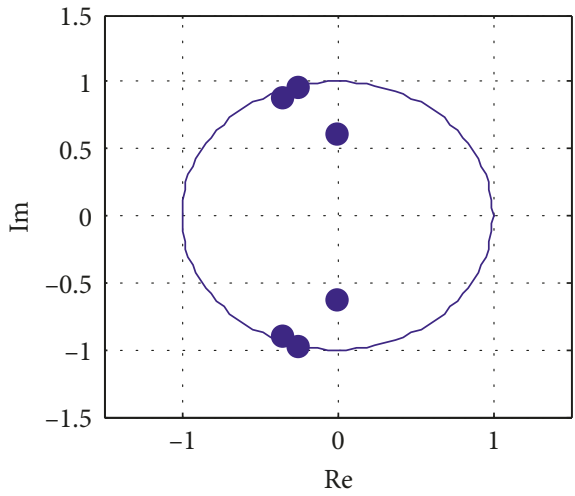

(b)

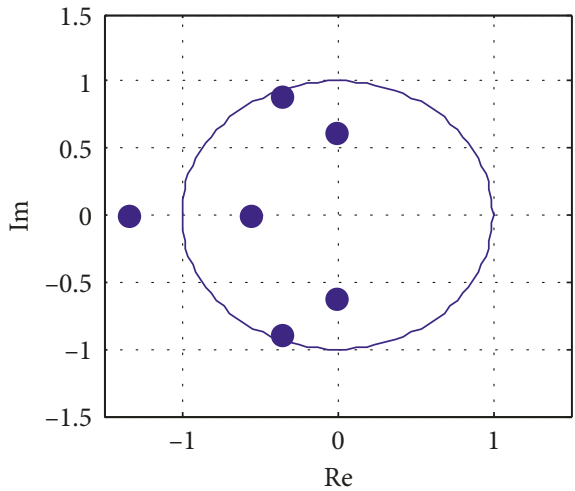

(d)

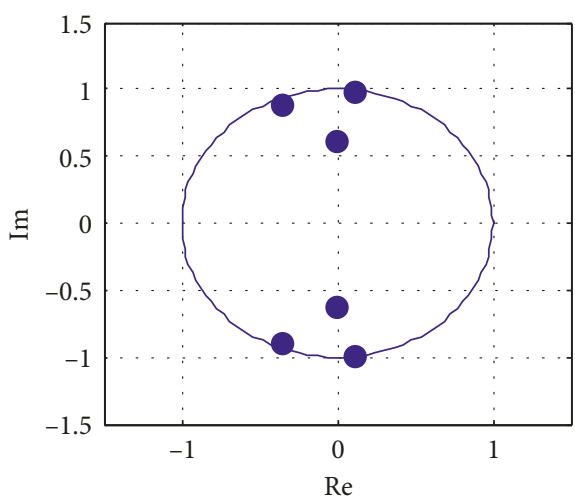

(f)

Figure 18: Position distribution of the maximum Floquet multiplier with pinion speed. (a) $\Omega=650 \mathrm{rpm}$. (b) $\Omega=1000 \mathrm{rpm}$. (c) $\Omega=$ $1450 \mathrm{rpm}$. (d) $\Omega=2500 \mathrm{rpm}$. (e) $\Omega=2250 \mathrm{rpm}$. (f) $\Omega=2850 \mathrm{rpm}$. 


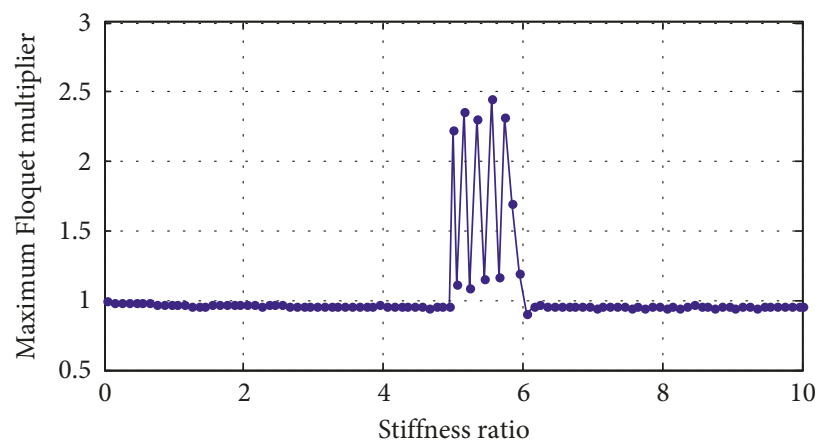

Figure 19: Diagram of the maximum Floquet multiplier with support stiffness ratio.

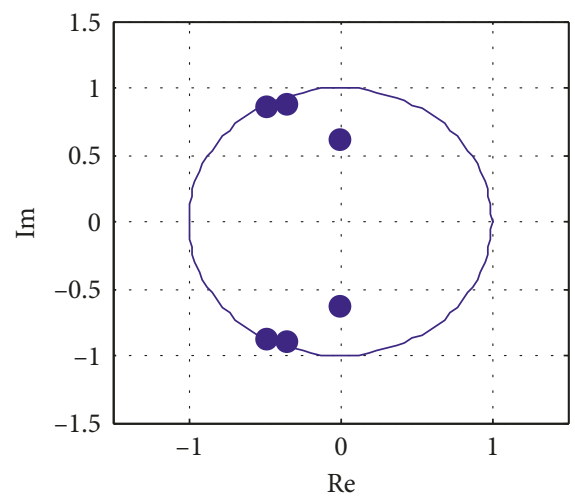

(a)

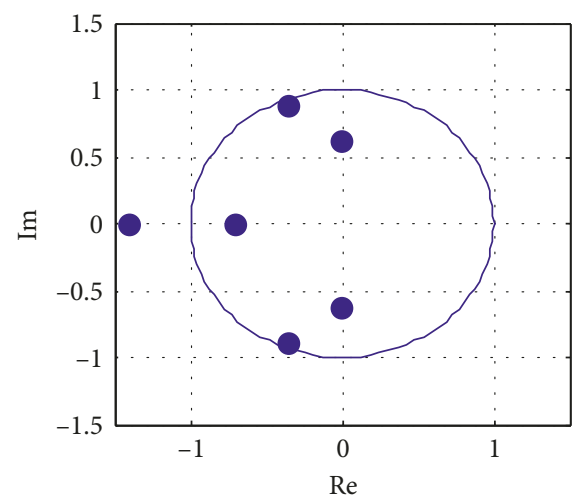

(c)

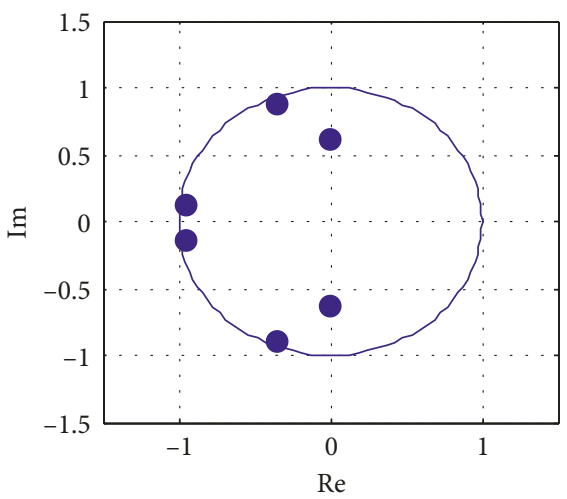

(b)

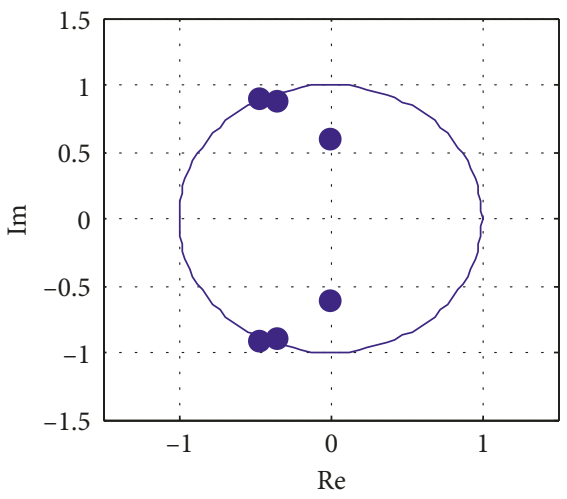

(d)

Figure 20: Position distribution of the maximum Floquet multiplier with support stiffness ratio. (a) $K=3$. (b) $K=4.7$. (c) $K=5.4$. (d) $K=5.9$.

4.3.2. Effect of Pinion Speed on Stability. The variation diagram of the maximum Floquet multipliers $\left|\lambda_{i}\right|$ against the pinion speed of the gear system is displayed in Figure 17. It can be seen that when $\Omega$ is less than $1160 \mathrm{rpm}$, $\left|\lambda_{i}\right|$ is less than 1, as shown in Figures 18(a) and 18(b), in which the maximum Floquet multipliers are in the unit circle, and the stable periodic solution is asymptotically stable according to the Floquet theory. But when the speed is changed at district of (1250-1270) or (1440-1830) rpm, the maximum Floquet multiplier is away from the unit circle in the form of a pair of conjugate complex eigenvalues. It means that the period-doubling bifurcation occurs, as shown in Figure 18(c).
When $\Omega$ is changed at the district of (1840-2110) rpm, $\left|\lambda_{i}\right|$ is less than 1 , and the gear system returns to stable periodic- 1 state. With the increasing pinion speed, $\left|\lambda_{i}\right|$ is greater than 1 , and the system leaves periodic-1 motion and enters chaos within (2410-2670) rpm, as shown in Figure 18(d). Once the speed is higher than $2680 \mathrm{rpm}$, the system leaves chaos and enters periodic- 2 and periodic- 1 motion, as shown in Figures 18(e) and 18(f).

4.3.3. Effect of Support Stiffness Ratio on Stability. The variation diagram of maximum Floquet multipliers $\left|\lambda_{i}\right|$ against the support stiffness ratio $K$ of the system is displayed 
in Figure 19. It can be seen that when $K$ is less than $5,\left|\lambda_{i}\right|$ is less than 1 , and the stable periodic solution of the system is asymptotically stable, as shown in Figures 20(a) and 20(b). With the increasing $K$, the system leaves periodic- 1 motion and enters chaotic motion at the district of $(5 \sim 6)$, in which the maximum Floquet multipliers are away from the unit circle in the form of complex eigenvalues, as shown in Figures $20(\mathrm{c})$ and $20(\mathrm{~d})$. Once $K$ is higher than $6,\left|\lambda_{i}\right|$ is less than 1 , and the gear system returns to synchronous vibration with period-1.

\section{Conclusions}

Taking the locomotive gear system as the object, the chaotic characteristics of the transmission system are analyzed when the pinion speed and stiffness are changed. At the same time, the parameter stability of the system and the evolution of periodic motion are studied based on the Floquet theory. The following conclusions can be drawn from the study:

(1) The system is relatively stable when the locomotive gear system runs at low speed; however, with the increasing speed of the traction motor (pinion), the responses of the gear system enter the unstable state of the chaotic motion through a variety of ways, which has an effect on the stable operation of the locomotive. Therefore, when the railway locomotive is operated, the speed of the traction motor can be increased selectively so that the transmission system can leave an unstable area of the speed as soon as possible. The analysis results show that the speed of the traction motor (pinion) is nearly $1500 \mathrm{rpm}$ (rated speed), which ensures stable and safe operation of locomotives.

(2) With the increase of the support stiffness ratio of the pinion to gear, the stability of the system will fluctuate. Considering the manufacturing cost, the pinion stiffness of the gear system can be selectively designed. For instance, when the stiffness is varied from $4.5 \times 10^{9}$ to $4.9 \times 10^{9} \mathrm{~N} / \mathrm{m}$, the gear system reaches the stable motion with period-1. Therefore, the interval above is the most suitable range of selection in the stiffness design of the transmission system.

\section{Data Availability}

The data used to support the findings of this study are included within the article.

\section{Conflicts of Interest}

The authors declare that they have no conflicts of interest.

\section{Acknowledgments}

This work was supported by the National Natural Science Foundation of China (No. 51475386) and National Program on Key Basic Research Project (973 Program, No. 2015CB654801).
The authors thank NSFC and NPKBRP for providing financial support.

\section{References}

[1] J. Wei, P. Gao, X. L. Hu, W. Sun, and J. Zeng, "Effects of dynamic transmission errors and vibration stability in helical gears," Journal of Mechanical Science and Technology, vol. 28, no. 6, pp. 2253-2262, 2014.

[2] J. Y. Wang, H. T. Wang, and L. X. Guo, "Analysis of effect of random perturbation on dynamic response of gear transmission system," Chaos, Solitons and Fractals, vol. 68, pp. 78-88, 2014.

[3] Z. Luo, X. Sun, and J. N. Fawcett, "Coupled torsional-lateralaxial vibration analysis of a geared shaft system using substructure synthesis," Mechanism and Machine Theory, vol. 31, no. 3, pp. 345-352, 1996.

[4] Y. S. Xiong, K. Huang, T. Wang, Q. Chen, and R. Xu, "Dynamic modelling and analysis of the microsegment gear," Shock and Vibration, vol. 2016, Article ID 9691647, 13 pages, 2016.

[5] Y. J. Zhang, Z. Meng, and Y. Z. Sun, "Dynamic modeling and chaotic analysis of gear transmission system in a braiding machine with or without random perturbation," Shock and Vibration, vol. 2016, Article ID 8457645, 12 pages, 2016.

[6] Y. W. Liu, Y. X. Zhu, K. G. Zhao, Z. H. Liang, and H. B. Lin, "Effect of clearance configuration on gear system dynamics," Advances in Mechanical Engineering, vol. 10, no. 4, pp. 1-9, 2018.

[7] L. Xiang, Y. Zhang, N. Gao, and J. T. Xing, "Nonlinear dynamics of a multistage gear transmission system with multiclearance," International Journal of Bifurcation and Chaos, vol. 28, no. 3, article 1850034, 2018.

[8] Q. B. Wang, B. Zhao, Y. Fu, X. G. Kong, and H. Ma, “An improved time-varying mesh stiffness model for helical gear pairs considering axial mesh force component," Mechanical Systems and Signal Processing, vol. 106, pp. 413-429, 2018.

[9] A. Al-shyyab and A. Kahraman, "Non-linear dynamic analysis of a multi-mesh gear train using multi-term harmonic balance method: sub-harmonic motions," Journal of Sound and Vibration, vol. 279, no. 1-2, pp. 417-451, 2005.

[10] Y. J. Shen, S. P. Yang, and X. D. Liu, "Nonlinear dynamic of a spur gear pair with time-varying stiffness and backlash based on incremental harmonic balance method," International Journal of Mechanical Sciences, vol. 48, no. 11, pp. 1256-1263, 2006.

[11] J. Wang, T. C. Lim, and M. F. Li, "Dynamics of a hypoid gear pair considering the effects of time-varying mesh parameters and backlash nonlinearity," Journal of Sound and Vibration, vol. 308, no. 1-2, pp. 302-329, 2007.

[12] H. Ma, M. J. Feng, Z. W. Li, R. J. Feng, and B. C. Wen, "Time-varying mesh characteristics of a spur gear pair considering the tip-fillet and friction," Meccanica, vol. 52, no. 7, pp. 1695-1709, 2017.

[13] Z. G. Chen and Y. M. Shao, "Dynamic simulation of spur gear with tooth root crack propagating along tooth width and crack depth," Engineering Failure Analysis, vol. 18, no. 8, pp. 2149-2164, 2011.

[14] X. H. Liang, M. J. Zuo, and T. H. Patel, "Evaluating the timevarying mesh stiffness of a planetary gear set using the potential energy," Proceedings of The Institution of Mechanical Engineers Part C: Journal of Mechanical Engineering Science, vol. 228, no. 3, pp. 535-547, 2014. 
[15] Z. G. Wan, H. R. Cao, Y. Y. Zi, W. P. He, and Y. M. Chen, "Mesh stiffness calculation using an accumulated integral potential energy method and dynamic analysis of helical gears," Mechanism and Machine Theory, vol. 92, pp. 447-463, 2015.

[16] J. Wei, P. Gao, X. L. Hu, W. Sun, and J. Zeng, "Effects of dynamic transmission errors and vibration stability in helical gears," Journal of Mechanical Science and Technology, vol. 28, no. 6, pp. 2253-2262, 2014.

[17] A. Farshidianfar and A. Saghafi, "Global bifurcation and chaos analysis in nonlinear vibration of spur gear systems," Nonlinear Dynamics, vol. 75, no. 4, pp. 783-806, 2014.

[18] A. P. Seyranian, F. Solem, and P. Pedersen, "Multi-Parmeter linear periodic systems sensitivity analysis and applications," Journal of Sound vibration, vol. 229, no. 1, pp. 89-111, 2000.

[19] X. Liu, S. Wang, and Y. Shen, "Nonlinear vibrations of 3-DOF geared rotor-bearing system," Mechanical Science and Technology, vol. 23, pp. 1191-1193, 2004.

[20] M. Vaishya and R. Singh, "Analysis of periodically varying gear mesh systems with Coulomb friction using Floquet theory," Journal of Sound and Vibration, vol. 243, no. 3, pp. 525-545, 2001.

[21] Y. Yao, H. J. Zhang, Y. M. Li, and S. H. Luo, "The dynamic study of locomotives under saturated adhesion," Vehicle System Dynamics, vol. 49, no. 8, pp. 1321-1338, 2011.

[22] H. Ma, R. Z. Song, X. Pang, and B. C. Wen, "Time-varying mesh stiffness calculation of cracked spur gears," Engineering Failure Analysis, vol. 44, pp. 179-194, 2014.

[23] H. Ma, J. Zeng, R. J. Feng, X. Pang, Q. B. Wang, and B. C. Wen, "Review on dynamics of cracked gear systems," Engineering Failure Analysis, vol. 55, pp. 224-245, 2015.

[24] H. Ma, J. Zeng, R. J. Feng, X. Pang, and B. C. Wen, "An improved analytical method for mesh stiffness calculation of spur gears with tip relief," Mechanism and Machine Theory, vol. 98, pp. 64-80, 2016.

[25] J. D. Wang, Numerical and experimental analysis of spur gears in mesh, Ph.D. thesis, Curtin University of Technology, Perth, Australia, 2003.

[26] A. Saxena, A. Parey, and M. Chouksey, "Time varying mesh stiffness calculation of spur gear pair considering sliding friction and spalling defects," Engineering Failure Analysis, vol. 70, pp. 200-211, 2016.

[27] F. Chaari, T. Fakhfakh, and M. Haddar, "Analytical modelling of spur gear tooth crack and influence on gear mesh stiffness," European Journal of Mechanics-A/Solids, vol. 28, no. 3, pp. 461-468, 2009.

[28] J. G. Wang, J. Z. Zhou, D. W. Dong, Y. Bin, and C. R. Huang, "Nonlinear dynamic analysis of a rub-impact rotor supported by oil film bearings," Archive of Applied Mechanics, vol. 83, no. 3, pp. 413-430, 2013. 


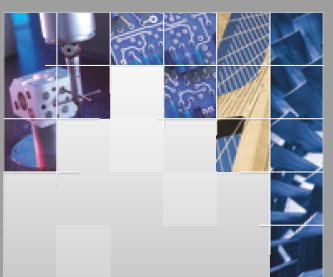

\section{Enfincering}
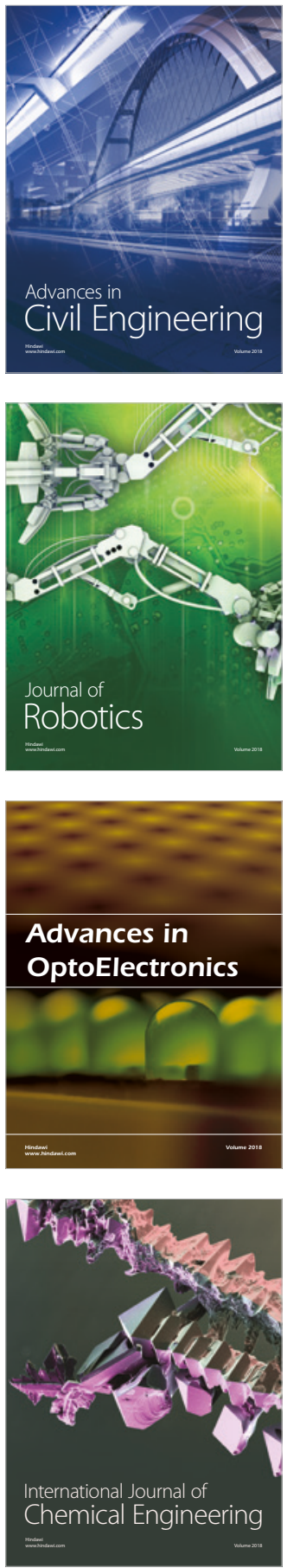

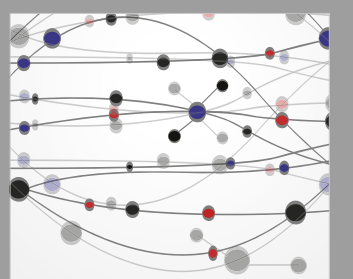

\section{Rotating \\ Machinery}

The Scientific World Journal

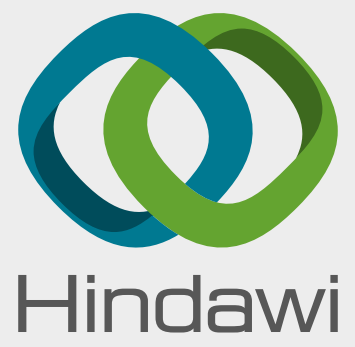

Submit your manuscripts at

www.hindawi.com
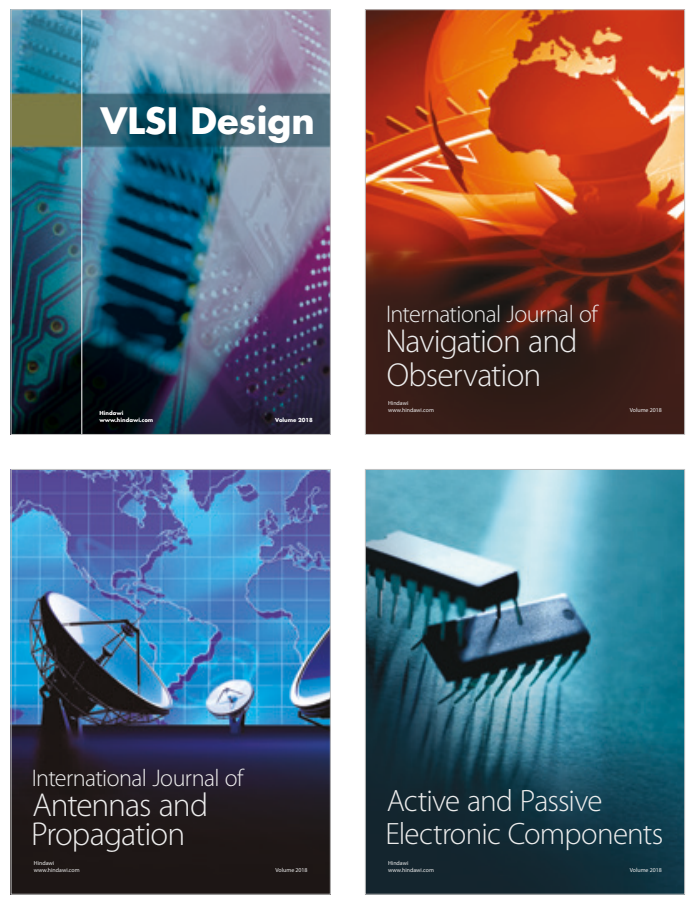
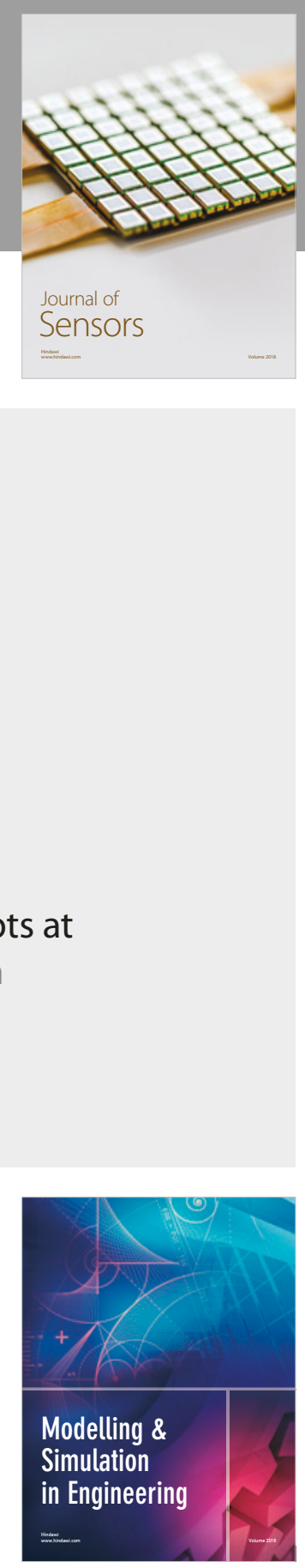

\section{Advances \\ Multimedia}
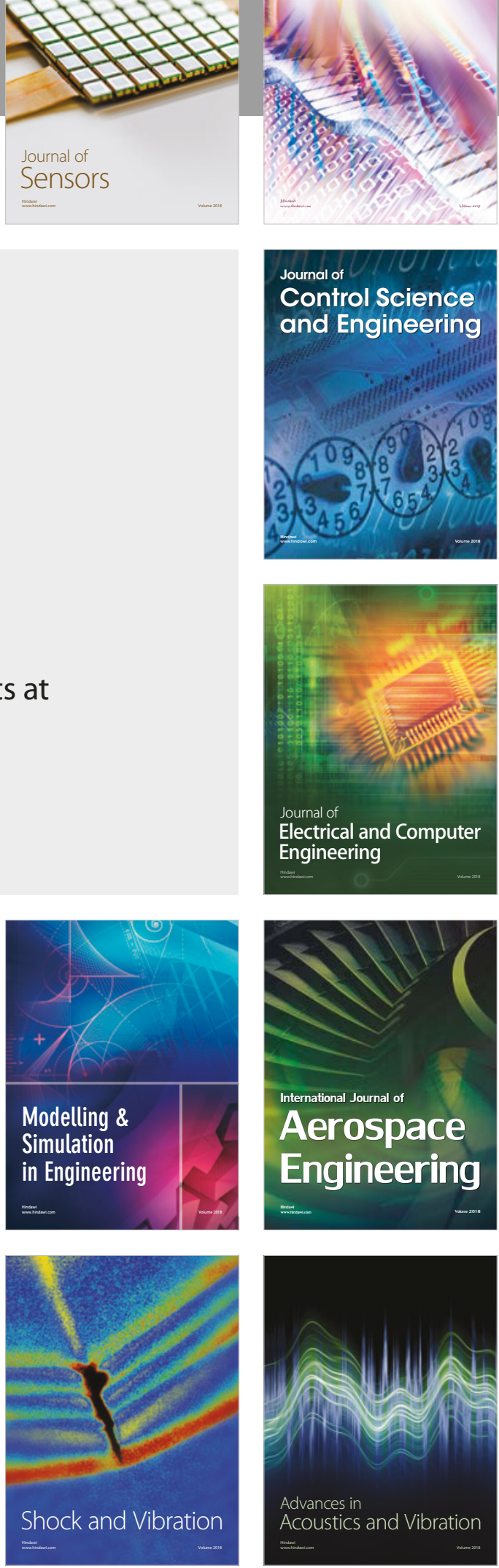